J. Clin. Chem. Clin. Biochem.

Vol. 14, 1976, pp. 429-431

\title{
Chromatographische Untersuchung des Substratverhaltens von 8-Brom-adenosin-5'-0-triphosphat gegenüber Nucleosiddiphosphatkinase
}

\author{
Von Gudrun Nagel ${ }^{1}$ ), Elke Schiller und E. Schlimme
}

Institut für Klin. Biochemie und Physiologische Chemie der Medizinischen Hochschule, Hannover und Laboratorium für Biol. Chemie der Universität - Gesamthochschule -, Paderborn

(Eingegangen am 6. April 1976)

Zusammenfassung: 8-Brom-ATP ist Substrat der Nucleosiddiphosphatkinase. Die Substratspezifität $\left(K_{\mathrm{m}}\right)$ des Analogons ist nicht signifikant verschieden vom unmodifizierten ATP. Die Spezifität des Enzyms ist nicht von der Substratkonformation abhängig.

Da das bei der Nucleosiddiphosphatkinase-Reaktion entstehende 8-Brom-ADP kein Substrat der Pyruvatkinase ist, ist der übliche optische Test im Falle dieses Analogons nicht anwendbar. Es wurde deshalb die ADP-Bildung der 'Nucleosiddiphosphatkinase-Reaktion chromatographisch verfolgt.

Chromatographic investigation of the substrate properties of 8-bromo-ATP in the nucleoside diphosphate kinase reaction

Summary: 8-bromo-ATP is a substrate of nucleoside diphosphate kinase. Its reactivity is similar to that of ATP, and the reaction appears to be unaffected by the different conformations of these two substrates.

The nucleoside diphosphate kinase reaction with 8-bromo-ATP cannot be monitored photometrically (optical test) using an NADH/NAD ${ }^{+}$-dependent enzyme system, because the product 8-bromo-ADP is not a substrate for pyruvate kinase. Measurements of the nucleoside diphosphate kinase reaction with 8-bromo-ATP therefore, were carried out by chromatographic analysis of the nucleoside-triphosphate/diphosphate ratio.

\section{Einleitung}

Nucleotidanaloga gilt neuerdings größere Aufmerksamkeit hinsichtlich ihrer therapeutischen Anwendbarkeit $(1,2)$. Experimentelle Untersuchungen über die Beteiligung von Analoga natürlich vorkommender Nucleotide an enzymatischen Reaktionen des Nucleotidstoffiwechsels liegen deshalb im klinisch-biochemischen Interesse.

Die vorliegende Arbeit befaßt sich mit den Eigenschaften eines Adenosin 5'-O-triphosphat (ATP):Analogons, dem 8-Brom-ATP, àls Phósphoryldonator gegenüuber Nucleosiddiphosphaten in der von der Nucleosiddiphosphatkinase katalysierten Phosphoryltransfer-Reaktion.

\section{Material und Methoden}

$\left[{ }^{14} \mathrm{C}\right] 8-\mathrm{Brom}-\mathrm{ATP}$ wurde aus $\left[{ }^{14} \mathrm{C}\right] \mathrm{ATP}(50 \mathrm{mCi} / \mathrm{l}, 196 \mathrm{Ci} / \mathrm{mol}$, Amersham-Buchler, Braunschweig) durch Bromieren in wäßriger Lösung hergestellt $(3,4)$. Die Aktivität der eingesetzten Nucleotidlösungen betrug: $\left[{ }^{14} \mathrm{C}\right] 8-\mathrm{Brom}-\mathrm{ATP} 1 \mu \mathrm{mol}=3,0 \times 10^{4} \mathrm{Imp} /$ $\left.\mathrm{min},{ }^{14} \mathrm{C}\right] \mathrm{ATP} 1 \mu \mathrm{mol}=9,7 \times 10^{5} \mathrm{Imp} / \mathrm{min}$. Die Reinheitskontrolle des ATP-Analogons erfolgte durch Liquid- $(5,6)$ und Dünnschichtchromatographie. Die Dünnschichtchromatographie wurde auf PËI-Cellulosefolien (Polygram CEL 300 PEI/UV, Macherey und Nagel, Düren) aufsteigend mit $0,75 \mathrm{~mol} / \mathrm{l}$ $\mathrm{KH}_{2} \mathrm{PO}_{4} \mathrm{pH} 4.1$ als Fließmittel ausgefuihrt. $\mathrm{R}_{\mathrm{f}}$-Werte: 8-Brom$\mathrm{ATP}=0,19 ; \mathrm{ATP}=0,25 ; 8$-Brom-ADP $=0,48 ; \mathrm{ADP}=0.59$, Nucleosiddiphosphatkinase aus Rinderleber (ATP: nucleoside diphosphate kinase EC $2.7 .4 .6 ; 5 \mathrm{mg} / \mathrm{ml}$, etwa 80 units $/ \mathrm{mg}$, Boehringer Biochemica, Mannheim). Radioaktivitätsmessungen wurden im Flüssigkeitsscintillationszähler (Packard Tricarb, Modell 544) ausgeführt.

\section{Ergebnisse und Diskussion}

Photometrisch wird der von der Nucleosiddiphosphatkinase katalysierte Phosphoryltransfer im Indikator:
1) Diese Arbeit enthält Errgebnisse der Med. Dissertation von G. $\mathbf{N}$. 
system a-c getestet (vgl. l.c. (7), (8), (9)):
a) ATP + dGDP
Nucleosiddiphosphatkinase
$\mathrm{ADP}+\mathrm{dGTP}$
b) ADP + Phosphoenolpyruvat
Pyruvatkinase
ATP + Pyruvat
c) Pyruvat $+\mathrm{NADH}+\mathrm{H}^{+}$
Lactatdehydrogenase
Lactat $+\mathrm{NAD}^{+}$

Wird 8-Brom-ATP in diesem Indikatorsystem auf seine Substrateigenschaften hin untersucht, so zeigt sich im optischen Test keine Reaktion. Es wird darüber hinaus in Anwesenheit von ATP eine kompetitive Hemmung der Gesamtreaktion a-c $\left(K_{\mathrm{i}}=400 \mu \mathrm{mol} / \mathrm{l}\right)$ durch das ATPAnalogon beobachtet. Diese Befunde konnten aufgrund chromatographischer Auftrennung der Ansätze der Reaktion b des Indikatorsystems zugeordnet werden. 8-Brom-ATP ist Substrat der Nucleosiddiphosphatkinase, das Reaktionsprodukt 8-Brom-ADP aber kein Substrat der Pyruvatkinase.
a) 8-Brom-ATP + dGDP
b) 8-Brom-ADP + Phosphoenolpyruvat

Aus den o. g. Gründen konnte das Substratverhalten von 8-Brom-ATP gegenüber der Nucleosiddiphosphatkinase im Reaktionsansatz nicht mit einem Indikatorsystem verfolgt werden, sondern mußte chromatographisch ausgewertet werden. Zur Überprüfung des chromatographischen Verfahrens wurde die Nucleosiddiphosphatkinase-Reaktion zunächst mit $\left[{ }^{14} \mathrm{C}\right]$ ATP untersucht. Der Reaktionsansatz enthielt: $300 \mu \mathrm{l} 0,25 \mathrm{~mol} / 1 \mathrm{Tris} / \mathrm{HCl}$

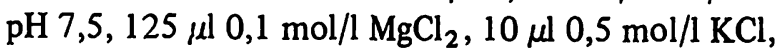
$10 \mu \mathrm{l} 0,03 \mathrm{~mol} / \mathrm{l} \mathrm{dGDP}, 2,5 \mu \mathrm{l}$ Nucleosiddiphosphatkinase. Die $\left[{ }^{14} \mathrm{C}\right] \mathrm{ATP}$-Konzentration variierte im Reaktionsansatz zwischen 0,11 und $1,7 \mathrm{mmol} / 1$. Die Reaktion wurde durch Zugabe von ATP gestartet. Innerhalb der ersten 20 Sekunden (linearer Bereich) nach dem Start der Reaktion wurden zu verschiedenen Zeitpunkten mehrere (4-5) Proben von je $50 \mu \mathrm{l}$ entnommen und in Eppendorfgefäßen, die $10 \mu \mathrm{l} 0,25 \mathrm{~mol} / \mathrm{l}$ ortho-Phosphorsäure enthielten, denaturiert. Die Start- und Entnahmezeit wurde auf einem Schreiber (Papiervorschub $250 \mathrm{~mm} / \mathrm{min}$ ) markiert. Aliquote Teile der denaturierten Proben wurden wie oben beschrieben aufgetrennt. Die Radioaktivität der [ $\left.{ }^{14} \mathrm{C}\right] \mathrm{ADP}$-Flecken wurde nach Ausschneiden aus der Dünnschichtchromatographie-Folie im Scintillationszähler gemessen. In entsprechender Weise wurden die Standardchromatogramme für $\left[{ }^{14} \mathrm{C}\right] \mathrm{ATP}$ bzw. $\left[{ }^{14} \mathrm{C}\right] \mathrm{ADP}$ ausgewertet.

Abbildung 1 zeigt das Lineweaver-Burk Diagramm für die Bestimmung der Michaelis-Menten Konstanten $K_{\mathrm{m}}$ für ATP. $K_{\mathrm{m}}($ ATP $\left.)=0,59(0,40-0,91)^{2}\right) \mathrm{mmol} / 1$ gegenüber $K_{\mathrm{m}}(\mathrm{ATP})=0,67 \mathrm{mmol} / \mathrm{lim}$ optischen Test (Literatur 2) Vertrauensbereich $95 \%$ für die graphische $K_{\mathrm{m}}$-Bestimmung,
vgl. dazu (10).
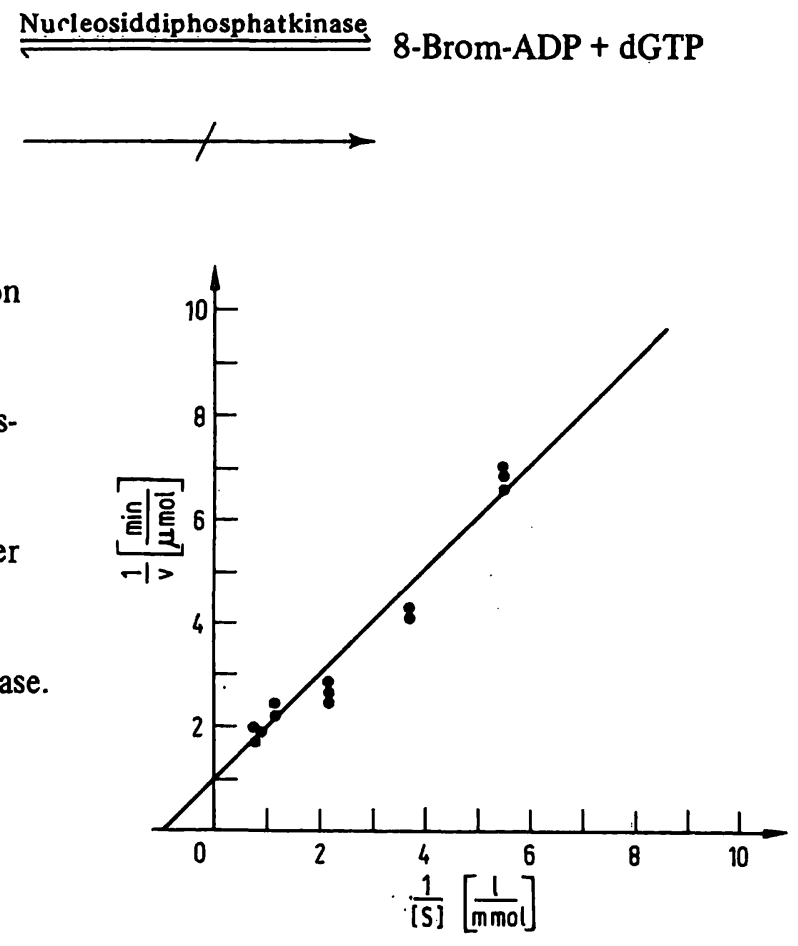

Abb. 1. Lineweaver-Burk-Diagramm für die Bestimmung von $K_{\mathrm{m}}$ (ATP).

$\left.y=0,89( \pm 0,13)^{2}\right) x+1,51( \pm 0,39) ; r=0,99$. $\left.K_{\mathrm{m}}(\mathrm{ATP})=0,59(0,40-0,91)^{2}\right) \mathrm{mmol} / \mathrm{l}$. $\left.V=0,66(0,53-0,98)^{2}\right) \mu \mathrm{mol} / \mathrm{min} \cdot$ Ansatz. $\left.52,8(42,4-71,2)^{2}\right) \mu \mathrm{mol} / \mathrm{min} \cdot \mathrm{mg}$ Nucleosiddiphosphatkinase.

(11): $\left.K_{\mathrm{m}}(\mathrm{ATP})=0,5 \mathrm{mmol} / \mathrm{l}\right)$. Entsprechend wurde wie für ATP beschrieben - das Substratverhalten von $\left[{ }^{14} \mathrm{C}\right] 8$-Brom-ATP untersucht: Die 8-Brom-ATP-Konzentration im Reaktionsansatz variierte zwischen 0,19 und $1,4 \mathrm{mmol} / \mathrm{l}$. Die experimentellen Befunde sind graphisch in Abbildung $2 \mathrm{im}$ Lineweaver-Burk Diagramm aufgetragen. Die Bestimmung der Michaelis-Menten Konstante ergab $K_{\mathrm{m}}(8$-Brom-ATP $\left.)=1,07(0,68-1,97)^{2}\right) \mathrm{mmol} / \mathrm{l}$. Die $K_{m}$ - und die $V$-Werte (vgl. Legende Abb. 1,2) des natürlichen und des chemisch modifizierten ATP-unterscheiden sich somit unter Zugrundelegung des o.a. Ver- 


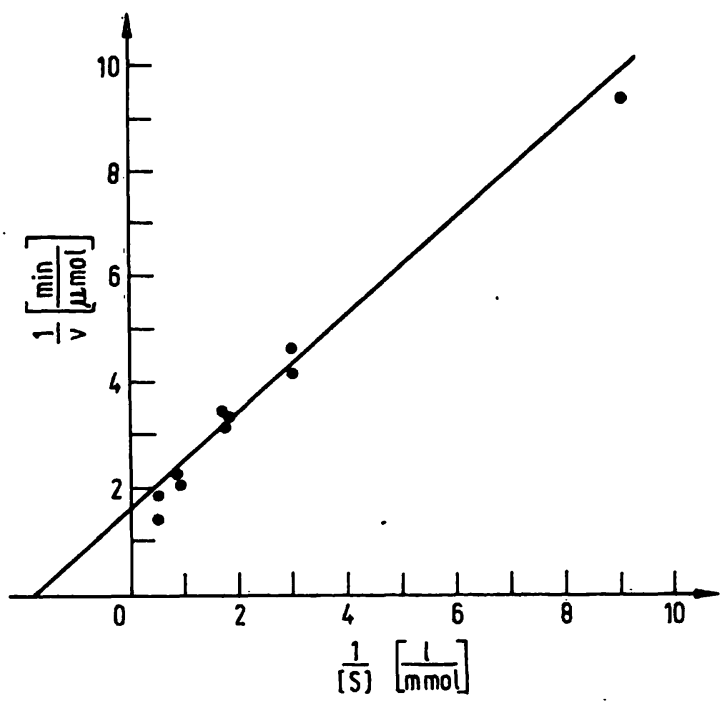

Abb. 2. Lineweaver-Burk-Diagramm für die Bestimmung von $K_{\mathrm{m}}$ (8-Brom-ATP).

$\left.y=1,03( \pm 0,13)^{2}\right) x+0,96( \pm 0,37) ; r=0,98$.

$K_{\mathrm{m}}(8-$ Brom-ATP $\left.)=1,07(0,68-1,97)^{2}\right) \mathrm{mmol} / \mathrm{l}$.

$\left.V=1,04(0,75-1,69)^{2}\right) \mu \mathrm{mol} / \mathrm{min} \cdot$ Ansatz.

$\left.=83,2(60,0-135,2)^{2}\right) \mu \mathrm{mol} / \mathrm{min} \cdot \mathrm{mg}$ Nucleosiddiphosphatkinase.
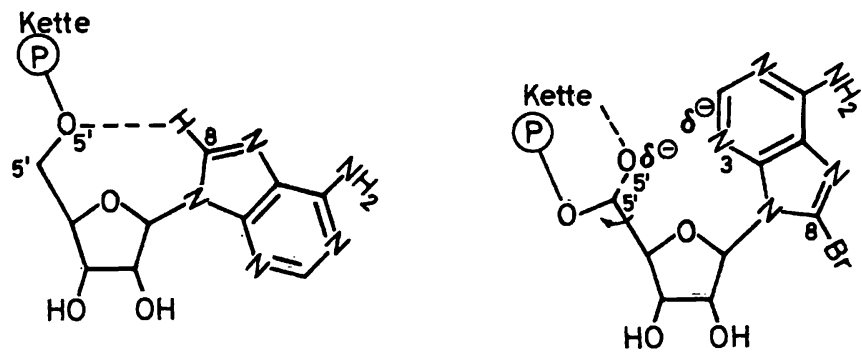

Abb. 3. a) Konformation des ATP. Stellung der Base: anti; Stellung der exocyclischen C4'-O5'-Gruppe: gauche, gauche.

b) Konformation des 8-Brom-ATP. Stellung der Base: syn; Stellung der exocyclischen C4'-O5'-Gruppe: gauche, trans.

\section{Literatur}

1. Cohen, S. S. (1975). Biochem. Pharmacol. 24, 1929-1932.

2. Mah, H. D. \& Daly, J. W. (1975). Biochim. Biophys. Acta 404, 49-56.

3. Reist, E. J., Calkins, D. F., Fisher, L. V. \& Goodman, L. (i968). J. Org. Chem. 33, 1600-1603.

4. Ikehara, M. \& Kesugi, S. (1969). Chem. Pharm. Bull 17, 348.

5. Stahl, K. W., Schlimme, E. \& Bojanovski, D. (1973). J. Chro mátogr. 83, 395-404.

6. Schlimme, E. \& Stahl, K. W. (1974). Hoppe Seyler's Z. Physiol. Chem. 355, 1139-1142.

7. Bergmeyer, H. U., Gawehn, K. \& Graß1, M. (1970), in Handbuch der Énzymatischen Analyse (Bergmeyer, H. U. ed.) Bd. I S. 449-450 Verlag Chemie, Weinheim.

8. Mourad, N. \& Parks jr., R. E. (1966). J. Biol. Chem. 241, $271=278$.

9. Chiga, M., Oda, A. \& Holtzer, R. L. (1963). Arch. Biochem. Biophys. 103, 366. trauensbereiches nicht signifikant, obwohl beide Nucleotide in Lösung in unterschiedlichen Konformationen (Abb. 3a, b) vorliegen. Im ATP nimmt die Nucleobase bevorzugt eine anti-Position um die $\mathrm{N}$-glykosidische Bindung ein (12-14), im bromierten ATP dagegen liegt der Adenin-Ring aus sterischen Gründen in der syn-Konformation vor (12-14). Mit der syn/anti-Konformation hängt die Position der exocyclischen $\mathrm{C5}^{\prime}$-O5'-Gruppe und damit die Stellung der Phosphatkette relativ zur Ribose eng zusammen. So ist die exocyclische $\mathrm{C5}^{\prime}-\mathrm{O5}^{\prime}$ Gruppe im ATP wegen der elektrostatischen Anziehung zwischen dem $\mathrm{C} 8 \mathrm{-H}$ und dem $\mathrm{O5}^{\prime}$ in einer gauche, gauchePosition stabilisiert, während im 8-Brom-ATP aus der syn-Konformation der Nucleobase eine elektrostatische Abstoßung zwischen dem N3 und dem O5' $^{\prime}$ resultiert, weshalb eine gauche, trans--Konformation bevorzugt ist (13-15). Im Gegensatz zu anderen Enzymen des Nucleotid- und Nucleinsäurestoffwechsels (16) sowie des mitochondrialen Adeninnucleotidtranslokators $(6,17)$ ist die Nucleosiddiphosphatkinase nicht konformationsspezifisch. Die biologische Funktion dieses Enzyms ist nicht an eine ungehinderte syn $\rightleftharpoons$ anti Isomerisierung des Nucleotids gebunden. 8-Brom-ATP kann somit ATP als Phosphoryldonator in der Nucleosiddiphosphatkinase-Reaktion ersetzen. In situ Messungen an der mitochondrialen Nucleosiddiphosphatkinase, die vorwiegend im mitochondrialen Intermembranraum lokalisiert ist (18), zeigten das gleiche Substratverhalten dieses ATP-Analogons.

\section{Danksagung}

Herrn Professor Lamprecht danken wir für sein förderndes Wohlwollen. Dem Landesamt für Forschung Nordrhein-Westfalen gilt unser Dank für finanzielle Unterstützung.

10. Stahl, K. W., Schlimme, E. \& Eckstein, F. (1974). FEBSLett. 40,241-246.

11. Glaze, R. P. \& Wadkins, C. L. (1967). J. Biol. Chem. 242, 2139-2150.

12. Saenger, W. (1973). Angew. Chem. Int. Ed. Engl. 16, 680-690.

13. Westhof, E., Röder, O., Croneiss, I. \& Lüdemann, D. H. (1975). Z. Naturforsch. 30c, 131-140.

14. Lee, C. H., Evans, F. E. \& Sarma, R. H. (1975). J. Biol. Chem. 250, 1290-1296.

15. Hruska, F. H. (1973) in Conformation of Biological Molecules and Polymers, (Bergmann, E. D. und Pullman, B. eds.) S. 345, Acad. Press, New York.

16. Kapuler, A. M., Monney, C. \& Michelson, A. M. (1970). Biochim. Biophys. Acta 217, 18-29.

17. Boos, K. S., Schlimme, E. Bojanovski, D. \& Lamprecht, W. (1975). Eur. J. Biochem. 60, 451-458.

18. Schnaitman, C. A. \& Greenawalt, J. W. (1968). J. Cell. Biol. $38,158-175$.
Prof. Dr. Dr. Eckhard Schlimme Laboratorium für Biol. Chemie im FB Naturwiss. der GH Pohlweg 55 D-4790 Paderborn 
